\title{
Design, Analysis, and Experimental Results of Micromachined Single-structure Triaxis Vibratory Gyroscope with Advanced Coupling Mechanism
}

\author{
Seyeong Seok, ${ }^{1}$ Sanghee Moon, ${ }^{2}$ Bumjoo Kim, ${ }^{3 *}$ and Geunbae Lim ${ }^{1 * *}$ \\ ${ }^{1}$ Department of Mechanical Engineering, Pohang University of Science and Technology, \\ 77 Cheongam-ro, Nam-gu, Pohang, Gyeongsangbuk-do 37673, Republic of Korea \\ ${ }^{2}$ Standing Egg Inc., Twosun World Building 7F, 221 Pangyoyeok-ro, \\ Bundanggu, Seongnam-si, Gyeonggi-do 13494, Republic of Korea \\ ${ }^{3}$ Department of Mechanical \& Automotive Engineering, Kongju National University, \\ 1223-24 Cheonan Daero, Seobuk-gu, Cheonan-si, Chungnam 31080, Republic of Korea
}

(Received June 7, 2018; accepted July 17, 2018)

Keywords: MEMS, gyroscope, simulation, prototype development

In this work, a novel micromachined monolithic triaxis gyroscope with an advanced anchor mechanism is designed and its structural characteristics are analyzed. Micromachined gyroscopes are usually packed in small packages, causing a high squeeze film damping effect that reduces the quality factor of out-of-plane vibration, resulting in lowered out-of-plane sensitivity. The proposed gyroscope has a four-mass single structure wherein the opposing masses vibrate in the opposite direction perpendicular to the direction they face, with the help of 'tree-shaped' coupling springs. The simulated driving and $x$-, $y$-, and $z$-axis sensing resonant frequencies are 19946, 20227, 20294, and $20361 \mathrm{~Hz}$, respectively. Also, the prototype of the gyroscope was fabricated and tested. It showed a driving $Q$-factor of 106 and a scale factor of 7 $\mathrm{mV} / \mathrm{deg} / \mathrm{s}$.

\section{Introduction}

As a result of the notable development of MEMS technologies over decades, micromachined inertial sensors are widely used because of their advantages in many applications over conventional bulk sensors, such as low cost, small size, and mass-producible fabrication. ${ }^{(1,2)}$

Most micromachined gyroscopes are vibratory ones that sense angular rotation by utilizing the Coriolis effect between the drive and sense modes. Early research usually focused on single- or two-axis gyroscopes. ${ }^{(1)}$ However, since most of today's user application devices have become compact and small, the sensors must also be accordingly small. The main approach to miniaturizing the sensor is to measure the angular rate of multiple axes with a sensor. There have been two different approaches to measuring three axes of the applied angular rate. ${ }^{(3)}$ One is to assemble three single-axis gyroscopes orthogonally, but the difficulty in orthogonal

\footnotetext{
*Corresponding author: e-mail: bumjoo@kongju.ac.kr

**Corresponding author: e-mail: limmems@postech.ac.kr

https://doi.org/10.18494/SAM.2018.2022
} 
assembly could cause misalignment that leads to reduced performance, increased packaging cost, and need for additional compensation control.

The other approach is a single-structure triaxis gyroscope. Despite its complex design requirements, it has many advantages over assembling multiple devices, such as integration cost, size reduction, and power consumption. Reported single-structure triaxis gyroscopes include tuning fork gyroscopes (TFGs) and vibratory wheel gyroscopes. STMicroelectronics reported a gyroscope with four connected proof masses that vibrate inward and outward together, two out-of-plane motions, and one in-plane motion that senses triaxis angular rate. The design and simulation of a vibratory wheel gyroscope were reported by Tsai and Fang, ${ }^{(4)}$ and later by Xia et al. ${ }^{(5)}$ One wheel-shaped proof mass vibrates along the $z$-axis, and the in-plane element senses the $z$-axis rate, while the $x$ - and $y$-axis rates are sensed by out-of-plane tilting.

One of the key issues of a micromachined triaxis monolithic gyroscope is cross-axis coupling. After decoupling structures between the drive and sense modes were reported, ${ }^{(6,7)}$ decoupling structures have become a must for micromachined gyroscopes. Since a singlemass triaxial gyroscope should have the degrees of freedom of multiple axes on one mass, a decoupling structure has been proposed by Sonmezoglu et al. as a way to avoid interference between the axes. ${ }^{(8)}$ The cross-axis coupling of a vibrating gyroscope can be roughly classified into two types. One is the interference between the drive and sense modes, or quadrature error, and the other is interference among the sense modes that occurs during angular rate detection, or cross-axis error.

In this paper, a novel micromachined triaxis vibratory gyroscope is proposed. A novel coupling structure that reduces stress and transfers vibration orthogonally between proof masses is presented. The decoupling structure placed between the drive and sense modes is shown to successfully suppress coupling.

\section{Working Principle}

The designed triaxis gyroscope structure is based on four connected masses with four degrees of freedom for three-axis angular rate sensing and has dimensions of $1700 \times 1700 \times 25$ $\mu \mathrm{m}^{3}$ as shown in Fig. 1. Four suspended masses, coupled by a 'tree-shaped' coupling spring, are actuated by comb drive electrodes and vibrate in antisymmetric motion, which resembles a vibrating wheel but with a linear displacement of proof masses. The opposing masses, unlike in the tuning fork type, do not move towards each other but vibrate in antiphase and antiparallel directions. ${ }^{(9)}$

\section{Structural Design}

\subsection{Resonant frequencies}

The resonant frequency of each mode is designed to have a small separation. Because the structure has a single mass, the sensor may malfunction owing to unwanted vibrations or erroneous output may occur if the resonant frequencies of all the vibration modes are matched. Also, the loosely matched mode is less sensitive to variations in $Q$ factor and thus is more robust 


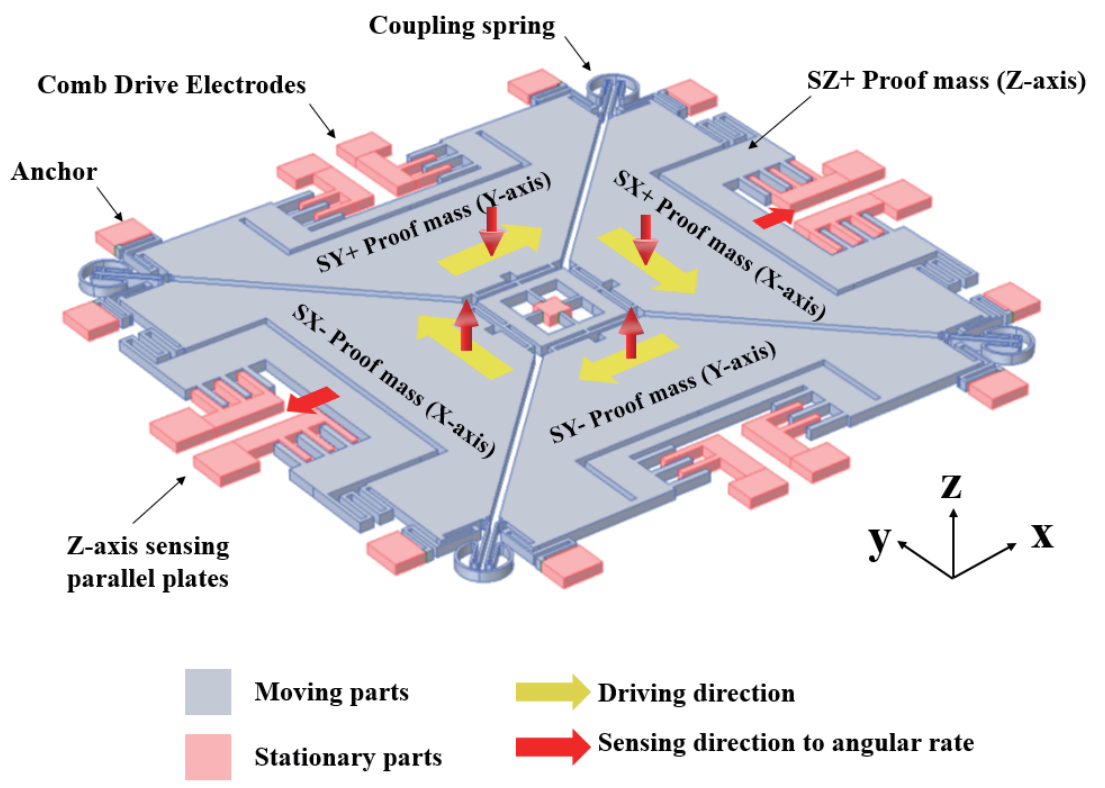

Fig. 1. (Color online) Simplified schematic of working principle of the gyroscope.

to error factors, such as temperature variations and fabrication errors. The resonant frequencies of the gyroscope are simulated using COMSOL Multiphysics and shown in Fig. 2. The resonant frequency differences between the modes are about $0.5 \%$.

\subsection{Decoupling in drive mode}

The drive mode excitation electrode structures are designed to be decoupled from the main proof masses to transmit only the movement in the drive direction. This driving mechanism leads to a loss in the driving amplitude of SY masses, about $8.1 \%$ according to the COMSOL simulation. However, other advantages include reduced parasitic levitation effects of driving electrodes $^{(10)}$ and cross-axis coupling with out-of-plane vibration modes. The out-of-plane vibration of the $z$-axis sensing electrode is reduced by $76.5 \%$ compared with the case without the decoupling structure, as shown in Fig. 3(b).

\subsection{Tree-shaped proof mass coupling springs}

The drive mode requires that all four masses have linear motion in the direction perpendicular to the adjacent mass. Since the driving force is directly applied to only two of the four masses, the driving force is transmitted to the remaining two through the coupling structure. This operation is realized through the unique tree-shaped mass connection spring located in the corner.

Table 1 shows that this tree-shaped spring offers up to $76 \%$-reduced off-axis displacement when driving, compared with linear, square, and circular coupling springs. The values were from the frequency response results when the driving displacements are equal to $1 \mu \mathrm{m}$. All 


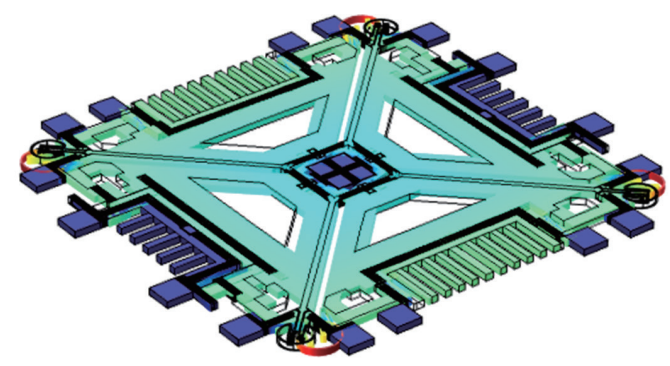

(a)

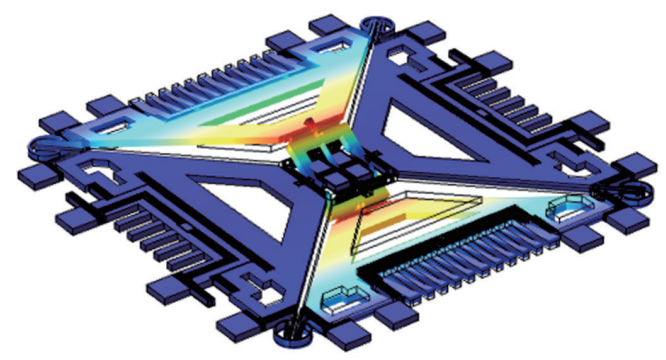

(c)

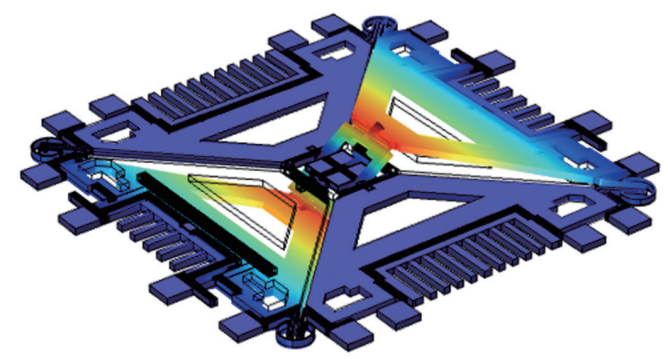

(b)

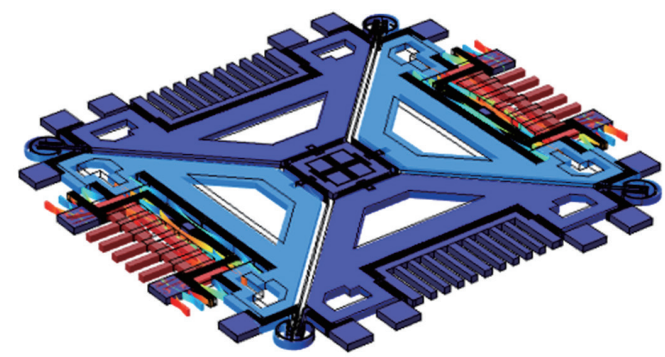

(d)

Fig. 2. (Color online) Resonant frequencies of drive and sense modes. (a) Drive mode at $19947 \mathrm{~Hz}$. (b) $X$-sense mode at $20227 \mathrm{~Hz}$. (c) $Y$-sense mode at $20294 \mathrm{~Hz}$. (d) Z-sense mode at $20361 \mathrm{~Hz}$.

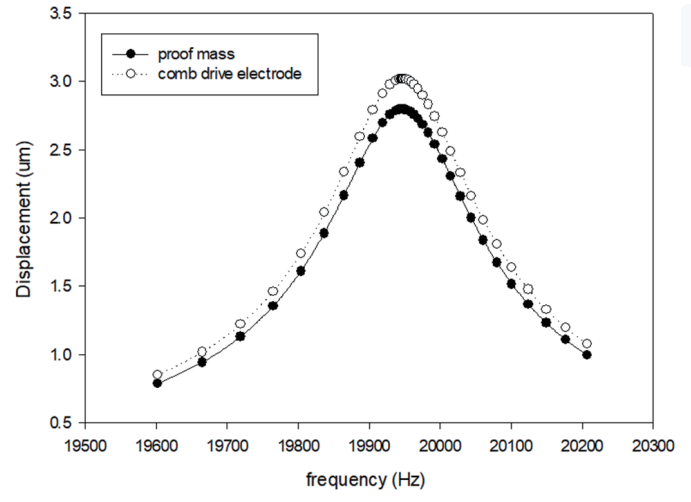

(a)

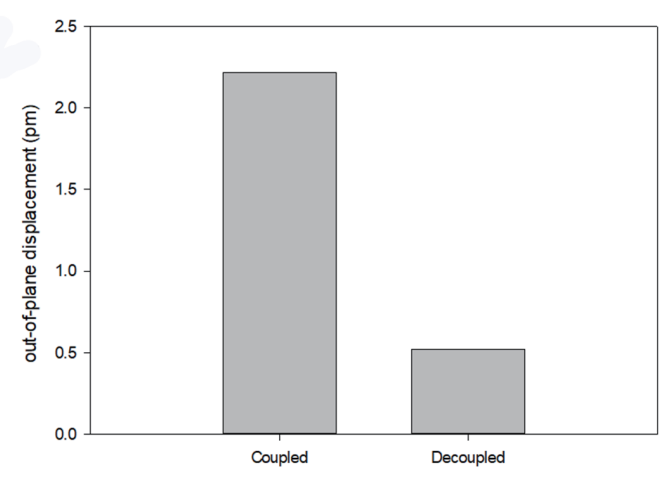

(b)

Fig. 3. (a) Driving displacement simulation results of comb drive electrodes and SY proof masses. (b) Effect of decoupling structure on out-of-plane displacement of comb drives with $1 \mathrm{deg} / \mathrm{s}$ applied angular rate along $y$-axis.

Table 1

Off-axis displacements and maximum von Mises stress on springs, with various coupling spring shapes.

\begin{tabular}{|c|c|c|c|c|}
\hline & Linear & Square & Circular & Tree-shape \\
\hline $\begin{array}{l}\text { Coupling spring } \\
\text { shape }\end{array}$ & & & & \\
\hline $\begin{array}{l}\text { Off-axis } \\
\text { disp. ratio }\end{array}$ & 0.3047 & 0.13813 & 0.1523 & 0.0731 \\
\hline $\begin{array}{l}\text { von Mises stress } \\
\left(\mathrm{kN} / \mathrm{m}^{2}\right)\end{array}$ & 366 & 665 & 538 & 47.5 \\
\hline
\end{tabular}


the coupling spring shapes are designed to fit in the $170 \times 170 \mu \mathrm{m}^{2}$ area. The beam width and thickness are 5 and $25 \mu \mathrm{m}$, respectively. Moreover, von Mises stress acting on the coupling spring is also greatly reduced by up to $93 \%$.

Through comparison with the other shapes, the role of each part of the tree-shaped spring can be predicted. The linear parts of the spring remain strong to aid in better transmission of vibration, while the rounded part deforms to reduce the stress on the spring and keeps the movements orthogonal to each other.

Since the driving force acts mainly on the SY masses and the motion is transmitted to the other masses via the tree-shaped coupling spring, a certain amount of structural loss inevitably occurs and the driving displacement of SX masses is reduced. The difference in displacement is about $7.6 \%$, but this difference can be compensated by loosely matching the resonant frequencies. The vibration frequency of the sense mode is equal to that of the drive mode. Therefore, when the modes are closely matched, the sense mode displacement will be greatly affected by $Q$-factor and vibration frequency differences. However, the closer the resonant frequency of the sense mode is from the resonant frequency of the drive mode, the larger the sensing displacement becomes.

The $x$-axis sense-mode resonant frequency is related to the SX mass having a relatively small driving displacement. As shown in Fig. 4, by setting the $x$-axis sense-mode resonant frequency closer to the drive-mode resonant frequency, it is possible to reduce the sense mode sensitivity difference from 7.3 to $0.7 \%$ despite the Coriolis force difference due to different driving displacements.

\subsection{Advantages of driving motion}

Intuitively, masses moving toward and away from each other should force nearby gas to contract and expand, resulting in squeeze film damping. However, the driving motion of the proposed gyroscope has advantages over the TFG-like motion in damping. When all four masses vibrate along the in-plane direction, the relative distance between masses is kept almost constant; it could dramatically reduce squeeze film damping and its nonlinear effects compared with when masses vibrate face-to-face. ${ }^{(11,12)}$

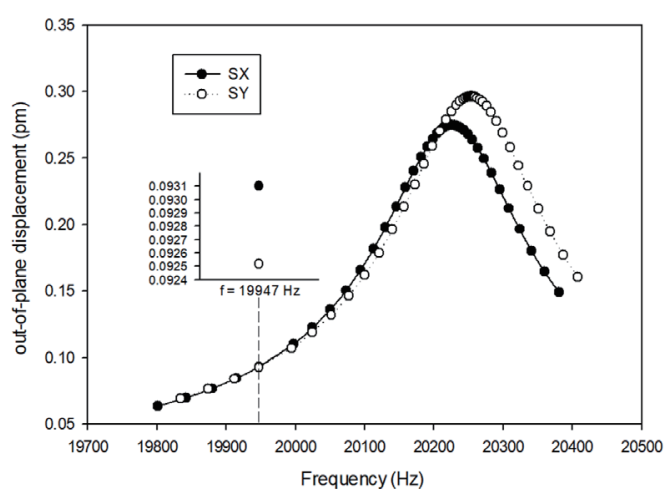

Fig. 4. Simulated displacements of out-of-plane sense modes with $1 \mathrm{deg} / \mathrm{s}$ applied angular rate. 


\section{Results and Discussion}

\subsection{Experimental setup}

To confirm the simulation results, a MEMS gyroscope with the same structure as our design was fabricated and tested with an analog circuit. The fabrication process and circuit diagrams are shown in Figs. 5 and 6, respectively. ${ }^{(13,14)}$ The gyroscope and circuit were tested with a rate table (1270VS-230, Ideal Aerosmith, USA) and the output data were obtained with a LabVIEW data acquisition system (PCIe-6363, NI, USA).

\subsection{Characteristics of gyroscope}

The resonant frequencies were measured at 22039, 22727, 22831, and $17241 \mathrm{kHz}$ for the drive mode and the $x$-, $y$-, and $z$-axis sense modes, respectively, and the drive mode $Q$-factor was measured to be 106 , as shown in Fig. 7. Considering that the $Q$-factor is low and all resonant frequencies are higher than the designed value, it is considered to be the influence

(a) mask patterning

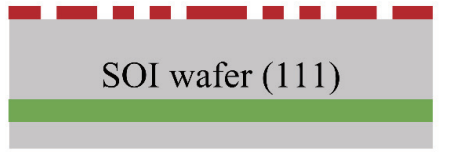

(b) Si etch
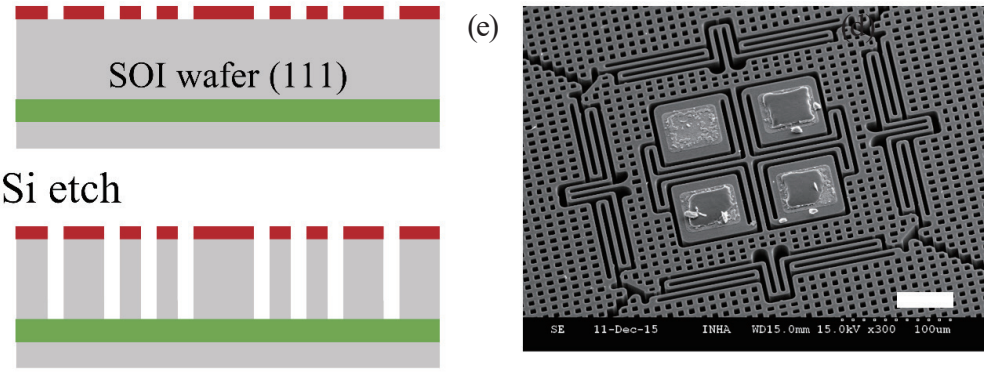

(c) side wall passivation

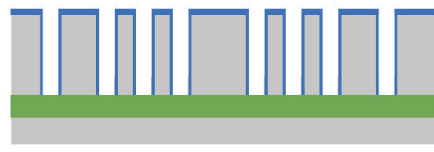

(f)

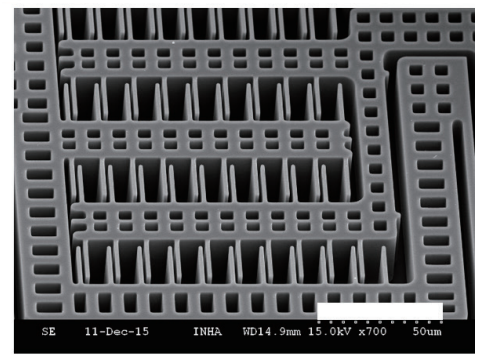

(d) oxide strip
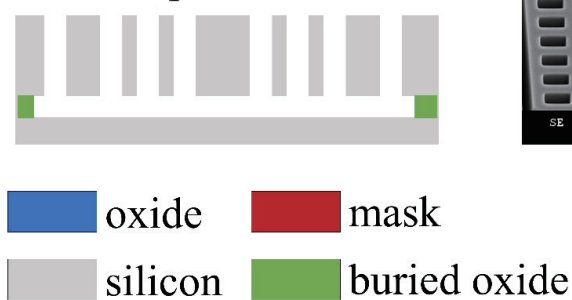

Fig. 5. (Color online) Gyroscope fabrication process and images of fabricated device. The prepared wafer is a (111)-oriented SOI wafer with a 25 - $\mu \mathrm{m}$-thick active layer and a 2 - $\mu \mathrm{m}$-thick buried oxide layer. (a) Mask patterning. (b) Anisotropic etching of silicon with deep reactive ion etch. (c) Side wall passivation with silicon oxide. (d) Oxide strip to release structure. (e) SEM image of center structures. (f) SEM image of variable gap capacitor. Scale bar = $50 \mu \mathrm{m}$. 


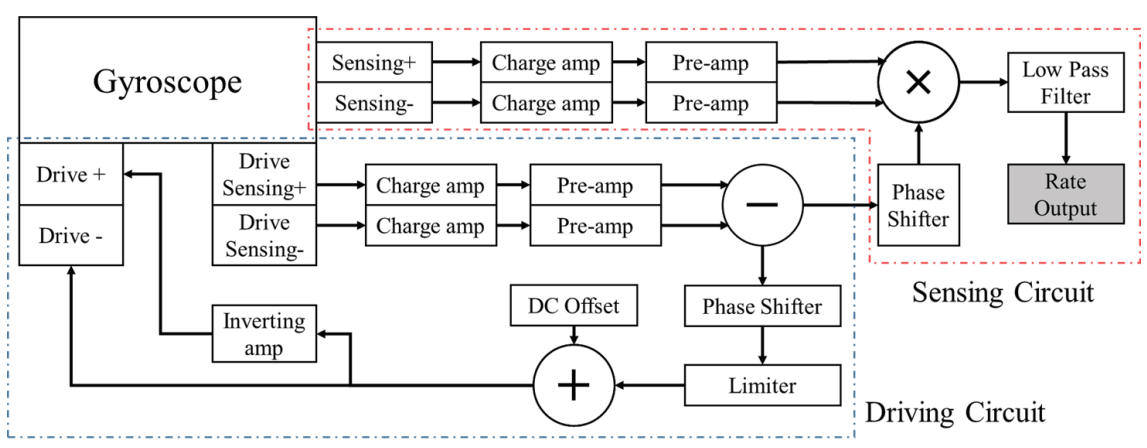

(a)

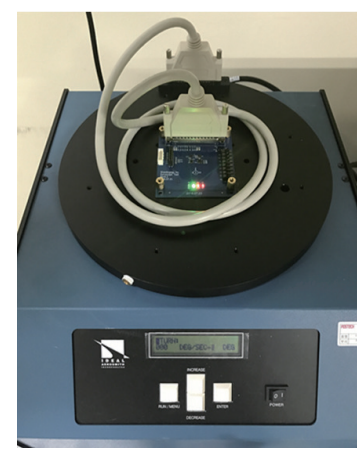

(b)

Fig. 6. (Color online) (a) Self-oscillation circuit and angular rate measuring circuit. (b) Gyroscope and circuit on rate table.

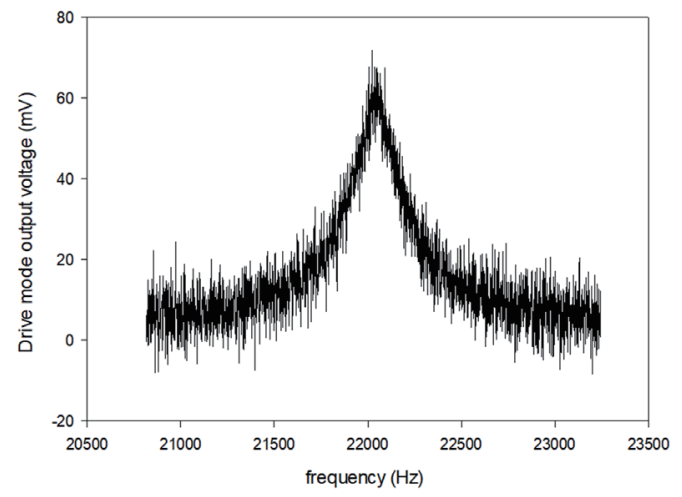

Fig. 7. Frequency sweep results of drive mode. The $Q$-factor was calculated to be about 106.

of the film damping of the air inside the structure owing to vacuum loss in the device. The resonant frequency of the $z$-axis differed greatly from those of the other axes. This low resonant frequency is highly likely the in-phase tuning-fork vibration. Because the $z$-axis sensing electrode structure is a gap variable capacitor with a parallel-plate structure, the influence of the damping on the $z$-axis sensing structure is greatly changed to affect the vibration motion. In addition, the parasitic capacitances of the $z$-axis differential sensing structures varied 1.5 times between each other that the differential sensing was nearly impossible. However, the device performance of the other two axes can be measured because the loosely matched design is robust to the $Q$-factor changes.

\subsection{Performance of gyroscope}

The angular rate output of the gyroscope was measured from -100 to $+100 \mathrm{deg} / \mathrm{s}$ with the rate table. The scale factor was measured to be about 7.55 and $6.88 \mathrm{mV} / \mathrm{deg} / \mathrm{s}$ for the $x$ - and $y$-axes, respectively (Fig. 8). The $z$-axis sensitivity could not be measured due to the differential sensing incompatibility. The $x$ - and $y$-axes were compared using the same circuit, and the sensitivity of the $x$-axis was measured to be slightly higher, different from what is predicted by 


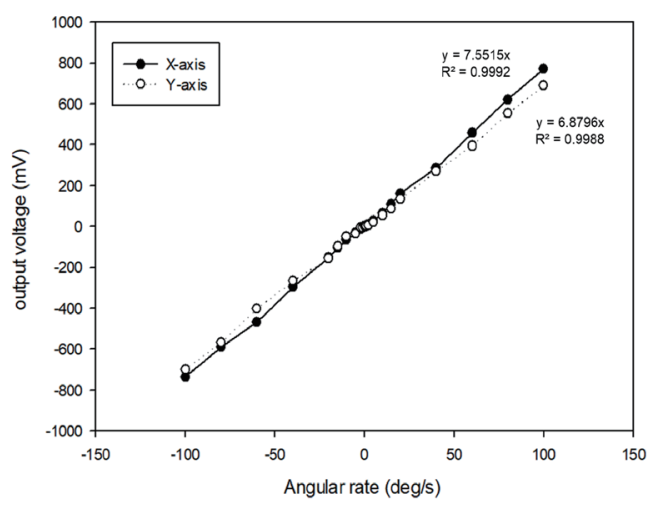

Fig. 8. Scale factor results for $x$ - and $y$-axes.

the simulation. This is because the resonant frequencies of the actual device are farther away from each other than in the design, such that the influence of the drive displacement itself is greater than that of the resonant frequency difference.

\section{Conclusions}

We proposed a novel triaxis MEMS vibrating gyroscope. The structure was designed to reduce film damping and cross-axis error. By connecting the proof masses using a unique treeshaped coupling spring, the proof mass vibrated linearly and the driving force was transmitted effectively. A decoupling structure for minimizing cross-axis error was also presented, and it could greatly reduce errors because of the precise parameter design. Thereafter, a prototype of the designed gyroscope was fabricated and evaluated. We successfully investigated the sensitivities of the $x$ - and $y$-axes, and confirmed that such a prototype can be used as a gyroscope. However, the whole three-axis performance was not measured owing to the vacuum loss in the fabrication process and the parasitic capacitance of sensing electrodes, and this remains as future work.

\section{Acknowledgments}

This work was supported by a National Research Foundation of Korea (NRF) grant funded by the Korea government (MSIP) (No. 2015R1A2A1A14027903).

\section{References}

1 K. Liu, W. Zhang, W. Chen, K. Li, F. Dai, F. Cui, X. Wu, G. Ma, and Q. Xiao: J. Micromech. Microeng. 19 (2009) 113001.

2 D. Xia, C. Yu, and L. Kong: Sensors 14 (2014) 1394.

3 D. Xia, L. Kong, and H. Gao: Sensors 15 (2015) 16929.

4 D. Tsai and W. Fang: Sens. Actuators, A $126(2006) 33$.

5 D. Xia, L. Kong, and H. Gao: Sensors 15 (2015) 28979.

6 W. Geiger, B. Folkmer, U. Sobe, H. Sandmaier, and W. Lang: Proc. Int. Conf. Solid State Sens. Actuator (Transducers, Chicago, 1997) 1129-1132. 
7 W. Geiger, W. U. Butt, A. Gaißer, J. Frech, M. Braxmaier, T. Link, A. Kohne, P. Nommensen, H. Sandmaier, W. Lang, and H. Sandmaier: Sens. Actuators, A 95 (2002) 239.

8 S. Sonmezoglu, P. Taheri-Tehrani, C. Valzasina, L. G. Falorni, S. Zerbini, S. Nitzan, and D. A. Horsley: IEEE Electron Device Lett. 36 (2015) 953.

9 S. Seok, S. Moon, K. Kim, S. Kim, S. Yang, and G. Lim: J. Sensor Sci. \& Tech. 26 (2017) 235.

10 C. Painter and A. Shkel: Proc. IEEE Sensors 2004 (IEEE Sensors, 2004) 508-511.

11 K. Jia: Retrieved from http://www.google.ch/patents/US9360319 (Accessed May 2018).

12 M. A. Shah, F. Iqbal, I. A. Shah, and B. Lee: J. Sensors 2016 (2016) 4615389.

13 J. Sung, J. Y. Kim, S. Seok, H. J. Kwon, M. Kim, G. Kim, and G. Lim: J. Micromech. Microeng. 24 (2014) 075013.

14 H. J. Kwon, S. Seok, and G. Lim: Sensors 17 (2017) 2663.

\section{About the Authors}

Seyeong Seok received his B.S. in 2010 and M.S. in 2012 from POSTECH in Korea, where he is currently a Ph.D. candidate in the department of Mechanical Engineering. His research interests include MEMS fabrication and micromachined inertial sensors. (athelas@postech.ac.kr)

Sanghee Moon received her B.S. degree from Chon-nam National University, Korea, in 2006 and is pursuing a Master's degree at Chung-Ang University. Since 2006, she has been a researcher at Standing Egg Inc. Her research interests are in MEMS Design, fabrication, and inertial sensors. (shmoon@standing-egg.co.kr)

Bumjoo Kim received his B.S. in 2005 and Ph.D. in 2013 from POSTECH in Korea. From 2013 to 2017, he worked at MIT, USA as postdoctoral associate. Since 2018, he has been an assistant professor at Kongju National University. His research interests are in MEMS application and microfluidics. (bumjoo@kongju.ac.kr)

Geunbae Lim received his B.S. and M.S. degrees from Youngnam University, Korea, in 1990 and 1992, respectively, and his Ph.D. degree from Tohoku University, Japan, in 1996. From 1996 to 2004, he worked in SAIT, Korea. Since 2004, he has been a professor at POSTECH. His research interests are in MEMS, BioMEMS, and sensors. (limmems@postech.ac.kr) 